\title{
A REVIEW OF RECENT RESEARCH IN INDOOR MODELLING \& MAPPING
}

\author{
M. Gunduz ${ }^{a}$, U. Isikdag ${ }^{b^{*}}$ M. Basaraner ${ }^{\mathrm{a}}$ \\ a Yildiz Technical University, Dept. of. Geomatic Engineering, Istanbul Turkey - memogedo@ gmail.com; mbasaran@yildiz.edu.tr \\ b Mimar Sinan Fine Arts University, Dept. of Informatics, Istanbul Turkey - uisikdag@ gmail.com
}

Commission IV, WG IV/7

KEY WORDS: Indoor, Mapping, Modelling, Navigation, IFC, CityGML, IndoorGML

\begin{abstract}
:
Indoor modeling and mapping has been an active area of research in last 20 years in order to tackle the problems related to positioning and tracking of people and objects indoors, and provides many opportunities for several domains ranging from emergency response to logistics in micro urban spaces. The outputs of recent research in the field have been presented in several scientific publications and events primarily related to spatial information science and technology. This paper summarizes the outputs of last 10 years of research on indoor modeling and mapping within a proper classification which covers 7 areas, i.e. Information Acquisition by Sensors, Model Definition, Model Integration, Indoor Positioning and LBS, Routing \& Navigation Methods, Augmented and Virtual Reality Applications, and Ethical Issues. Finally, the paper outlines the current and future research directions and concluding remarks.
\end{abstract}

\section{INTRODUCTION}

Our complex world requires developing much smarter scenarios for our living environments. Along with the availability of spatial information almost ubiquitously in the current societies, spatial applications have been expanding into indoor spaces. 3D spatial information about indoor environments has increasingly been demanded in various applications such as risk and disaster management, human trajectory identification, and facility management. The scientific and technological progress in 3D spatial data acquisition as well as 3D city and building modeling has been evolving into more sophisticated hardware, software, standards, techniques and uses specific to indoor modeling and mapping. Sensors provide a key role for acquisition of indoor information, hard sensors such as laser scanners, RGB-D cameras, and gyro sensors/accelerometers are today main subjects of research in the field, while there are opportunities that are provided by machine learning (i.e. selflearning devices that can be used as soft-sensors). Indoor Data Models such as IFC, CityGML and IndoorGML appear as the main focus of current research in the field. The studies in the area concentrate on enhancing these models and tailoring these models to fit the needs of different application domains. Another area that the paper is focusing on is Integration, i.e. a topic which includes studies on fusion of information coming from multiple sources and the area also covers efforts on integration of information residing in multiple data models. Another area of research in the field is indoor positioning which refers to the task of inferring the location of a mobile device inside a building. Although many techniques for positioning are available, a limited number of algorithms and methods can infer indoor location information from measurements. For indoor location-based services (LBS), both sufficiently accurate positioning as well as quality and information-rich indoor maps are required (Werner, 2014) Proposing new and better approaches for indoor navigation and routing is also an active field of research. In this field some studies focus on optimization of routing where there are dynamically changing conditions and multi-targets. Outputs of indoor modelling and navigation research facilitate the everyday operations of several domains including sales in retail stores, fire response scenarios, building maintenance activities. Virtual and augmented reality applications increase human's perception of real world and carry remarkable potential for indoor environments. Ethical issues also are important elements of research as these are concerned with privacy of people.

\section{METHODOLOGY}

The aim of the research was to review of literature in the field of Indoor Modelling and Mapping, and outlining the main research directions in this field. The study started with the review of literature focused on papers presented in ISPRS 3DGeoInfo Conference in last ten years and also covered the papers of Indoor 3D Workshop which has been organized in Cape Town in 2013. The researchers have come across 51 papers directly related to Indoor Modeling and Navigation among the papers that were investigated during the review. The findings of the review are then classified into 7 categories as A. Information Acquisition by Sensors, B. Model Definition, C. Model Integration, D. Indoor Positioning and LBS, E. Routing \& Navigation Methods, F. Augmented and Virtual Reality Applications, and G. Ethical Issues. The following section elaborates on these categories.

Corresponding author 


\section{RESEARCH FIELDS IN INDOOR MODELING}

\subsection{Information Acquisition by Sensors}

Sensors can be used to acquire information in order to build up the indoor models (i.e. such as laser scanners) and they also aid in locating people and objects within the indoor environment. Terrestrial laser scanners can be used for creating point clouds to represent indoor environment. In fact, this process is time consuming and requires detailed planning. The research has also demonstrated the use of Laser Scanners for localization. In fact, previous research indicates that only one mobile laser scanner is not sufficient for precise localization. Using two mobile laser scanners is required for achieving good localization results (Kaijaluoto et al., 2015). Point clouds can help in generation of the building models at different level of details (Achille and Fassi, 2006). Sometimes it may be hard to find 3D CAD models of buildings. Even it is possible to find the related $\mathrm{CAD}$ model, due to lack of the coordinate information they can be difficult to use. Besides, indoor objects' place may change or new objects may be added in time in the buildings. So, it could be helpful to scan, model the buildings with $3 \mathrm{D}$ terrestrial laser scanner with some intervals. However, it is time consuming to create 3D models and as the approach is not fully automated, and as it requires an experienced operator (Dongzhen et al., 2009). Fusion of sensor information is also found useful in information structuring. For example, Wang and Sohn (2010) used Airbone laser scanning (ALS) for outdoor information acquisition and terrestrial laser scanning (TLS) is used for indoor information acquisition. Architectural plans were then integrated with TLS data using point matching method. Finally, outdoor model and indoor model structured and semantically integrated simultaneously in the study. Acquired data from sensors can be beneficial in generation of semantically rich digital building models (Meouche et al., 2013) and models such as BIM. Yoon et al. (2015) utilized point clouds generated by laser scanners to create $3 \mathrm{D}$ geometries which would form the basis for a BIM. Stereo Vision Systems can also be used to acquire information about indoors. For instance, researchers have reported a portable system for seamless outdoor-indoor modeling which has four cameras and can be moved/operated by one person. In this system, four cameras gather stereo images and as a result 3D models can be automatically generated with high efficiency. (Shao et al., 2015). Sensors that acquire indoor information can also be used in aiding navigation for instance RGB-D cameras (Kanai et al., 2015) and commercial ones such as Microsoft Kinect were used for aiding indoor navigation. (Pagliari et al., 2015). It is also possible to generate point clouds from video recordings. Teo (2015) aimed to generate point clouds from video data. The phases of the process were camera calibration, video conversion and alignment, orientation modeling, dense matching and evaluation. Huge computational time, low image quality caused by video compression and motion blur were the weak sides of this method.

\subsection{Model Definition}

Building up data models for representing indoors is an important area of research in indoor modeling. Development and implementation of new data types and data structures is one of the key areas of model definition research. For example, Gold et al. (2006) implemented a Quad-Edge structure used for generating building exteriors. Boguslawski and Gold (2009) used Augmented Quad Edge (AQE) which is an irregular decomposition $3 \mathrm{D}$ data model. AQE is suitable to create primal and dual spaces simultaneously. Boguslawski and Gold (2010) stated that building models can be constructed using Euler operators. In their implementation states/attributes of node, edge, face or volume can be changed if needed. Multi-layered indoor models is an important research topic in the field. As indicated by Becker et al. (2009) in a multi-layered indoor model topographic and sensor space can be represented in different layers. While topographic space represents building's 3D model; stories, rooms, corridors and their relationships, sensor space represents Wi-Fi, RFID sensors and so on. International standards such as CityGML also provide indoor representations. CityGML Indoor Application Domain Extension (ADE) is an indoor spatial model (XML schema) based on CityGML. The ADE includes indoor space features and indoor facility features for indoor facility management (Kim et al., 2014). Shape grammars can also be used to define indoor models. For instance, Becker et al. (2013) proposed a full automatic approach to generate $3 \mathrm{D}$ building interior models from partly erroneous or incomplete observation data.

\subsection{Model Integration}

The topic of model integration covers integration of information acquired from sensors and also fusion of information coming from different models. As an example of the former method Wohlfeil et al. (2013) utilized multi-scale sensor systems and semi-global matching for reconstruction of cultural heritage sites. Nakagawa et al. (2015) proposed a methodology for generation of 3D topologies from indoor mobile LIDAR data. Fusion of information was viewed as a key method in instance (object) population in indoor models. For example, Isikdag and Zlatanova (2009) offer a formal framework for seamless integration between IFC and CityGML. Most of the model integration studies are focused on unidirectional information transfer, where information from IFC model is transferred into the CityGML model. The work of El-Mekawy et al. (2011) proposed a bidirectional data model integration for IFC and CityGML. In another effort by Laat and vanBerlo (2011) implemented a CityGML extension namely GeoBIM for extending CityGML with more detailed (semantic) information about the inner structure of buildings. Studies focusing on seamless integration of outdoor and indoor models and applications have also gained momentum recently. In this context, standards have a prominent role and some missing elements in relevant standards such as IFC and CityGML for indoor applications, particularly indoor navigation, are now being addressed by IndoorGML (IndoorGML Web, 2016). The more challenging issue is the development of standards for indoor location detection/computation technologies. This may continue to be a brake on the development of seamless indoor/outdoor location applications (UN-GGIM, 2015). Recent studies such as Kim and Lee (2015), Jung and Lee (2015) proposed methods for generation of IndoorGML objects. Internet of Things (IoT) is a new research field that focuses on enabling interaction between online "Things". Isikdag (2014) stated that integration of information from "Things" with indoor information models will provide unique opportunities for indoor localization and navigation. Indoor navigation models can be enriched by information coming from multiple resources. Information transfer from BIM into indoor navigation models is the mainstream approach in the field. Hijazi et al. (2010) built up indoor utility networks based on information derived from IFC BIMs. Geiger et al. (2014) proposed a method of generalization to represent BIM information in CityGML. Further efforts in this direction can contribute to the population of indoor model entities. In fact, as BIMs contain much detailed 
semantic and geometric information, information simplification is also required in this transformation. (Tang et al., 2015)

\subsection{Indoor Positioning and LBS}

There are many ways for indoor positioning like WiFi, RFID, Visible Light Connection and indoor GPS. Krishnamurthy (2015) gives a systematic classification of indoor positioning technologies (RF-based and non-RF-based) and methods (Figure 1). Under the RF-based technologies, it is common to employ Wi-Fi as the technology of choice, although Bluetooth, RFID, and cell phone technologies (cellular) are also possible choices. Among non-RF-based technologies, acoustic technologies that use either ultrasound or sound for localization have received attention. Dead reckoning and signage are the obvious choices for localization in indoor areas without a positioning infrastructure. Some combination of the approaches is also feasible. The common methodologies are those based on proximity to a known device, those that use the time of arrival (TOA) of a signal, those that use TOA and direction of arrival (DOA), and finally those that employ location fingerprinting. In terms of positioning, indoor areas have some disadvantages in comparison to outdoor environments. Weak signal penetration and multipath signal propagation make it difficult to determine the time or direction of arrival of signals. Besides, positional accuracy requirement is higher, usually at meter level or better. Furthermore, buildings are in most cases multi-storey and positioning method and localization schemes can not work accurately if they do not recognize this information.

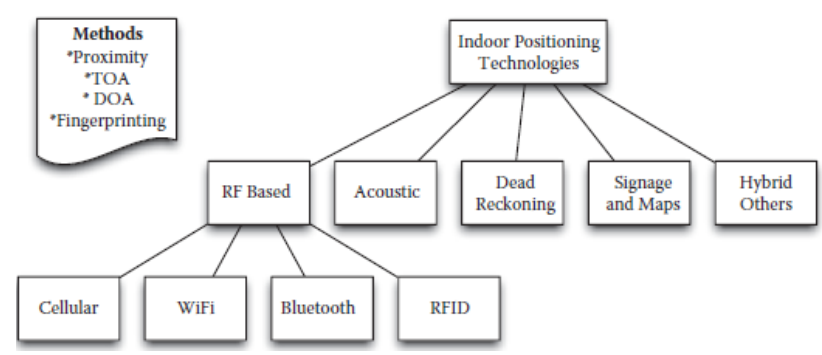

Figure 1. Positioning technologies and methods for indoor areas (Adapted from Krishnamurthy, 2015).

Integrated positioning system (IPS) used for more accurate inertial navigation. IPS hardware has a pair of stereo camera to acquire stereo images. (Grießbach et al., 2013). Fuse and Matsumoto (2015) proposed an information fusion methodology combining information coming from images, GPS, gyro, accelerometers and magnetic field sensors for localization. Lai et al., (2015) used low cost and readily available sensors calibrated and used for estimate step length and strength. Pedestrian navigation is possible by multi-sensor (accelerometer and gyroscope) fusion and fuzzy logic estimation. Ogawa et al. (2015) proposed an Indoor Messaging System (IMES) which is GPS like positioning system and Open Location Services (OpenLS) standard selected. Werner (2014) gives a schematic representation of building blocks of indoor location-based services (LBSs) (Figure 2). Indoor LBSs require precise geometric and semantic representation of buildings in addition to sufficiently accurate indoor positioning. It is easy to find geographic data sets and maps for outdoor environments but difficult to say the same for indoor spaces, which require $3 \mathrm{D}$ (or 2.5) maps to allow routing and navigation between floors (Karimi, 2015).

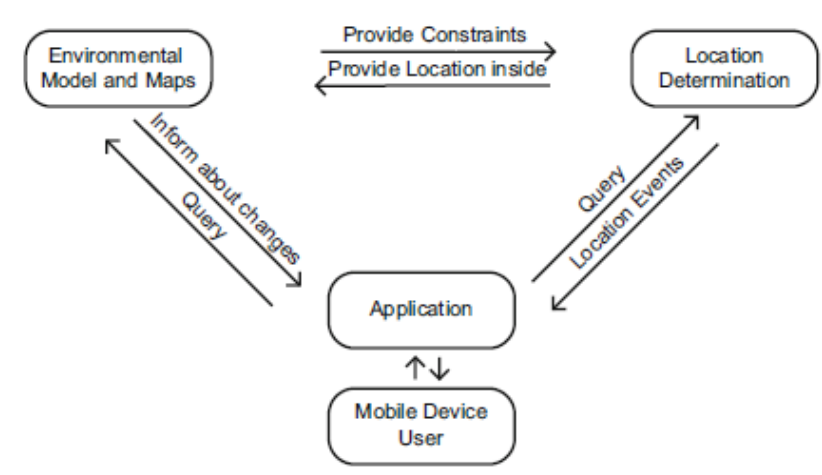

Figure 2. Building blocks of indoor location-based services (Adapted from Werner, 2014).

\subsection{Routing \& Navigation Methods}

Methods for facilitating routing and navigation in buildings is another valuable topic of indoor modeling research. As outlined by Karas et al. (2006) derivation of network models is a necessity to calculate the optimal routes for indoor navigation. Indoor navigation calculations mainly rely on shortest path algorithm, for instance Xiong et al. (2015) proposed a method that uses $\mathrm{A}^{*}$ algorithm to create $3 \mathrm{D}$ indoor paths. In fact, shortest path is not always the optimal one. For example, in a fire emergency situation, shortest path can be generated via blocking the paths where fire is ongoing but that is not enough to decide that path is secure enough. More parameters should be used to generate optimal route via risk analysis (Vanclooster et al., 2013). As mentioned by Atila et al. (2012) most of the indoor routing systems are based on $2 \mathrm{D}$ or $2,5 \mathrm{D}$ models. There are many algorithms for outdoor path routing, but indoor routing algorithms are not so diverse. Dijkstra's shortest path algorithm is being used at most of the researches. Cognitive indoor route algorithms are important and needed as much as outdoor routing, especially in three-dimensional systems. At the work of Vanclooster et al. (2013), Grum's least risk path algorithm for outdoor space is implemented to indoor routing. Using landmarks and salient images while explaining routes to other people is a common human behavior but indoor navigation systems don't have such semantics for this purpose. The research of Arendholz and Becker (2015) identified potential landmarks, signs and images and then classified them by the requirements. Finally, a thematic framework for navigation in existing building models like CityGML, IFC or $\mathrm{KML}$ is generated. People use a sign system to find their routes in Netherlands. Makri et al. (2015) proposed an indoor navigation system is similar with this sign system. A method used for generating graph model automatically. Floor signs created with this graph model. Most of the routing systems are not interactive; users can't change, modify or customize the network model. Khan et al. (2014) combined Multi-layered Space Event Model (MLSEM) and IndoorGML structures and proposed a context aware route planning cloud-based system. Work of Goetz and Zipf (2011) focused on navigation in more complex situations like considering obstacles at interior space, districted areas or vertical building parts. One way paths such as escalators or passport control points were also considered in their research. Weighted indoor routing graph which can generate different routes for disabled or elderly people was proposed for user adaptive routing. In order to facilitate seamless navigation indoors/outdoors, topological models representing connected indoor/outdoor spaces is required 
(Slingsby and Raper, 2008). Evacuation management is one of the primary domains which makes use of indoor routing and navigation methods. For instance, Atila et al. (2014) proposed a solution (data and network generation, visualization and evacuation model) for fire evacuation scenarios. In a disaster scenario, routing for shortest exit path in order to evacuation may not enough. People may rush to common doors and confluence may happen. Research of Choi and Lee (2009) aimed to calculate temporal changes during evacuation with proposed agent-based model.

\subsection{Augmented and Virtual Reality Applications}

There are currently no standards or best practices of how to display indoor navigation information. Several attempts use two-dimensional floor plans or three-dimensional building models and plot the current position, plus the routing information. There seems to be no formal investigation, if these representations are understood by the users and help the users in their navigational tasks (Krisp et al., 2015). As new way of communicating indoor spatial information, Augmented and Virtual Reality (AR\&VR) applications are used to assist user's wayfinding activities indoors. With the advent of new AR\&VR technologies such as Google Glass ${ }^{\mathrm{TM}}$, Microsoft HoloLens ${ }^{\mathrm{TM}}$, Occulus Rift ${ }^{\mathrm{TM}}$ and Samsung Gear ${ }^{\mathrm{TM}}$, various methodologies for utilizing these technologies will also be proposed and implemented by the researchers in coming years. For instance, a way-finding assistance system developed recently, provides an augmented reality based solution for pedestrians. The system generates coarse 3D model of destination feature from images and displays it at screen on the real-time video if the angle of the camera is right. Generated 3D model's size and view angle changes based on the distance and digital compass, gyroscope data acquired from the mobile device (Yokoi et al., 2015). Another implementation example is provided by Lee and Tsai (2015) where 3D building model from panoramic images were derived semi-automatically to assist AR based navigation.

\subsection{Ethical Issues}

The issues related to personal privacy and traceability have always been a major topic of indoor modeling and navigation related research. Verbree et al. (2013) discusses this issue in their paper "To Localise or to Be Localised with WiFi in The Hubei Museum?". Indoor positioning systems enable to track the position of people, and this help companies to send commercial messages depending on the location of them. In fact, as one could consider an unrelated content as spam, the person may feel uncomfortable when he/she received such a message. In order to prevent that perception, location based push message system was offered by Nakagawa (2013). A virtual geofence system created for this purpose with movement tracking. If the subject person stops/waits on a pre-determined location, the system considers that the subject is showing attention to that location and a push message content related to that location will then be sent to the user.

\section{CONCLUSION}

Spatial data collection, representation, processing, analysis and visualization methods for indoor modeling and mapping are still under development although significant progress has recently been made from scientific and technological perspective. Conferences such as ISPRS 3DGeoInfo and Indoor 3D forms nice academic forums for discussion of research related to indoor modelling and mapping. This paper has presented a systematic summary of the research outputs belonging to last ten years. Precise positioning techniques, sensor network systems, IoT, integrated indoor and outdoor data representation models and standards, more sophisticated LBS applications and advanced visualization techniques will be key research topics in indoor modeling and mapping research in coming years.

\section{REFERENCES}

Achille, C., Fassi, F., 2006. 3D multi-scale modelling of the interior of the Real Villa of Monza (ITALY). Innov. 3D Geo Inf. Syst. 195-205.

Arendholz, K., Becker, T., Arendholz, K., Becker, Á.T., Becker, T., 2015. Requirements on Building Models Enabling the Guidance in a Navigation Scenario Using Cognitive Concepts. Lect. Notes Geoinf. Cartogr. 157-173.

Atila, U., Karas, I.R., Rahman, A.A., Atila, U., Karas, I.R., Rahman, A.A., 2013. A 3D-GIS Implementation for Realizing 3D Network Analysis and Routing Simulation for Evacuation Purpose. Lect. Notes Geoinf. Cartogr. 249-260.

Atila, U., Karas, I.R., Turan, M.K., Rahman, A.A., 2014. Automatic Generation of 3D Networks in CityGML and Design of an Intelligent Individual Evacuation Model for Building Fires Within the Scope of 3D GIS. Innovations in $3 D$ GeoInformation Sciences. Springer International Publishing, pp. 123-142.

Becker, S., Peter, M., Fritsch, D., Philipp, D., Baier, P., Dibak, C., 2013. Combined Grammar for the Modeling of Building Interiors. ISPRS Ann. Photogramm. Remote Sens. Spat. Inf. Sci. II-4/W1, 1-6.

Becker, T., Nagel, C., Kolbe, T.H., 2009. A Multilayered Space-Event Model for Navigation in Indoor Spaces. 3D GeoInformation Sciences. Springer Berlin Heidelberg, Berlin, Heidelberg, pp. 61-77.

Boguslawski, P., Gold, C., 2009. Construction Operators for Modelling 3D Objects and Dual Navigation Structures. 3D Geo-Information Sciences. Springer Berlin Heidelberg, Berlin, Heidelberg, pp. 47-59.

Boguslawski, P., Gold, C., 2011. Rapid modelling of complex building interiors. Adv. 3D Geo-Information Sci., pp. 43-56.

Choi, J., Lee, J., 2009. 3D Geo-Network for Agent-based Building Evacuation Simulation. 3D Geo-Information Sciences. Springer Berlin Heidelberg, Berlin, Heidelberg, pp. 283-299.

Dongzhen, J., Khoon, T.Y., Zheng, Z., Qi, Z., 2009. Indoor 3D Modeling and Visualization with a 3D Terrestrial Laser Scanner. 3D Geo-Information Sciences. Springer Berlin Heidelberg, Berlin, Heidelberg, pp. 247-255.

El Meouche, R., Rezoug, M., Hijazi, I., Maes, D., 2013. Automatic Reconstruction of 3D Building Models from Terrestrial Laser Scanner Data. ISPRS Ann. Photogramm. Remote Sens. Spat. Inf. Sci. II-4/W1, 7-12.

El-Mekawy, M., Östman, A., Shahzad, K., 2011. Towards Interoperating CityGML and IFC Building Models: A Unified Model Based Approach. Adv. 3D Geo-Information Sci. XXXVIII, 73-93. 
Fuse, T., Matsumoto, K., 2015. Self-Localization Method By Integrating Sensors. ISPRS - Int. Arch. Photogramm. Remote Sens. Spat. Inf. Sci. XL-4/W5, 87-92.

Geiger, A., Benner, J., Haefele, K.H., 2015. Generalization of 3D IFC Building Models. 3D Geoinformation Science. Springer International Publishing, pp. 19-36.

Goetz, M., Zipf, A., 2011. Formal definition of a user-adaptive and length-optimal routing graph for complex indoor environments. Geo-spatial Inf. Sci. 14, 119-128.

Gold, C., Tse, R., Ledoux, H., 2006. Building Reconstruction - Outside and In. Innovations in 3D Geo Information Systems. Springer Berlin Heidelberg, Berlin, Heidelberg, pp. 355-369.

Grießbach, D., Baumbach, D., Börner, A., Zuev, S., 2013. Accuracy evaluation of stereo vision aided inertial navigation for indoor environments. Int. Arch. Photogramm. Remote Sens. Spat. Inf. Sci. - ISPRS Arch. 40, 13-17.

Hijazi, I., Ehlers, M., Zlatanova, S., Becker, T., Berlo, L. Van, 2011. Initial Investigations for Modeling Interior Utilities Within 3D Geo Context: Transforming Ifc- Interior Utility To Citygml / Utilitynetworkade. Advances in 3D Geo-Information Sciences, 95-113.

IndoorGML [WWW Document], 2016. URL http://www.opengeospatial.org/standards/indoorgml

Isikdag, U., 2014. A RESTful Service for Linking Sensors and Cellular Spaces. Innov. 3D Geo-Information Sci. 93-105.

Isikdag, U., Zlatanova, S., 2009. Towards Defining a Framework for Automatic Generation of Buildings in CityGML Using Building Information Models. 3D Geo-Information Sciences. Springer Berlin Heidelberg, Berlin, Heidelberg, pp. 79-96.

Jung, H., Lee, J., 2015. Indoor Subspacing To Implement Indoorgml for Indoor Navigation. ISPRS - Int. Arch. Photogramm. Remote Sens. Spat. Inf. Sci. XL-2/W4, 25-27.

Kaijaluoto, R., Hyyppä, A., 2015. Precise Indoor Localization for Mobile Laser Scanner. ISPRS - Int. Arch. Photogramm. Remote Sens. Spat. Inf. Sci. XL-4/W5, 1-6.

Kanai, S., Hatakeyama, R., Date, H., 2015. Improvement of 3D Monte Carlo Localization Using a Depth Camera and Terrestrial Laser Scanner. ISPRS - Int. Arch. Photogramm. Remote Sens. Spat. Inf. Sci. XL-4/W5, 61-66.

Karas, I.R., Batuk, F., Akay, A.E., Baz, I., 2006. Automatically Extracting 3D Models and Network Analysis for Indoors. Innovations in 3D Geo Information Systems. Springer Berlin Heidelberg, Berlin, Heidelberg, pp. 395-404.

Karimi, H.A., 2015. Indoor Wayfinding and Navigation. CRC Press, Inc. Boca Raton, FL, USA.

Khan, A., Yao, Z., Kolbe, T., 2015. Context Aware Indoor Route Planning Using Semantic 3D Building Models with Cloud Computing. 3D Geoinformation Science. Springer International Publishing, pp. 175-192.
Kim, M., Lee, J., 2015. Developing a Method to Generate Indoorgml Data From the Omni-Directional Image. ISPRS - Int. Arch. Photogramm. Remote Sens. Spat. Inf. Sci. XL-2/W4, 1719.

Kim, Y., Kang, H., Lee, J., 2014. Developing CityGML Indoor $\mathrm{ADE}$ to Manage Indoor Facilities. Innovations in $3 D$ GeoInformation Sciences. Springer International Publishing, pp. 243-265.

Krishnamurthy, P., 2015. Technologies for Positioning in Indoor Areas. Indoor Wayfinding and Navigation. CRC Press, Inc., FL, USA, pp. 35-51.

Krisp J. M., Jahnke M., Lyu H. and Fackler F., 2013. Visualization and Communication of Indoor Routing. Progress in Location-Based Services 2014. Springer International Publishing, pp. $33-44$.

Laat, R. De, Berlo, L. Van, 2011. Integration of BIM and GIS: The development of the CityGML GeoBIM extension. Adv. 3D Geo-Information Sci. 211-225.

Lai, Y.C., Chang, C.C., Tsai, C.M., Lin, S.Y., Huang, S.C., 2015. Development of a Pedestrian Indoor Navigation System Based on Multi-Sensor Fusion and Fuzzy Logic Estimation Algorithms. ISPRS - Int. Arch. Photogramm. Remote Sens. Spat. Inf. Sci. XL-4/W5, 81-86.

Lee, I.-C., Tsai, F., 2015. Applications of Panoramic Images: From $720^{\circ}$ Panorama To Interior 3D Models of Augmented Reality. ISPRS - Int. Arch. Photogramm. Remote Sens. Spat. Inf. Sci. XL-4/W5, 189-192.

Makri, A., Zlatanova, S., Verbree, E., Delaunay, C., 2015. an Approach for Indoor Wayfinding Replicating Main Principles of an Outdoor Navigation System for Cyclists. ISPRS - Int. Arch. Photogramm. Remote Sens. Spat. Inf. Sci. XL, 21-22.

Nakagawa, M., 2013. Improvement in the geofencing service interface using indoor positioning systems and mobile sensors. Int. Arch. Photogramm. Remote Sens. Spat. Inf. Sci. - ISPRS Arch. 40, 27-30.

Nakagawa, M., Yamamoto, T., Tanaka, S., Shiozaki, M., Ohhashi, T., 2015. Topological 3d Modeling Using Indoor Mobile Lidar Data. Int. Arch. Photogramm. Remote Sens. Spat. Inf. Sci. - ISPRS, 13-18.

Norris, J., Ordnance Survey, 2015. Future Trends in geospatial information management: the five to ten year vision, Second ed. Ogawa, K., Verbree, E., Zlatanova, S., Kohtake, N., Ohkami, Y., 2011. Toward seamless indoor-outdoor applications: Developing stakeholder-oriented location-based services. Geospatial Inf. Sci. 14, 109-118.

Pagliari, D., Cazzaniga, N.E., Pinto, L., 2015. Use of Assisted Photogrammetry for Indoor and Outdoor Navigation Purposes. ISPRS - Int. Arch. Photogramm. Remote Sens. Spat. Inf. Sci. XL-4/W5, 113-118.

Shao, Z., Li, C., Zhong, S., Liu, B., Jiang, H., Wen, X., 2015. 3D Reconstruction of Cultural Tourism Attractions From Indoor To Outdoor Based on Portable Four-Camera Stereo Vision System. ISPRS - Int. Arch. Photogramm. Remote Sens. Spat. Inf. Sci. XL-4/W5, 193-197. 
Slingsby, A., Raper, J., 2008. Navigable Space in 3D City Models for Pedestrians Brief Review of 3D city modelling approaches. Advances in 3D Geoinformation Systems. 49-64.

Tang, S.J., Zhu, Q., Wang, W.W., Zhang, Y.T., 2015. Automatic Topology Derivation From Ifc Building Model for in-Door Intelligent Navigation. ISPRS - Int. Arch. Photogramm. Remote Sens. Spat. Inf. Sci. XL-4/W5, 7-11.

Teo, T., 2015. Video-Based Point Cloud Generation Using Multiple Action Cameras. ISPRS - Int. Arch. Photogramm. Remote Sens. Spat. Inf. Sci. XL-4/W5, 55-60.

Vanclooster, a., Viaene, P., Van de Weghe, N., Fack, V., De Maeyer, P., 2013. Analyzing the applicability of the least risk path algorithm in indoor space. ISPRS Ann. Photogramm. Remote Sens. Spat. Inf. Sci. II-4/W1, 19-26.

Vanclooster, a., Viaene, P., Van de Weghe, N., Fack, V., De Maeyer, P., 2013. Analyzing the applicability of the least risk path algorithm in indoor space. ISPRS Ann. Photogramm. Remote Sens. Spat. Inf. Sci. II-4/W1, 19-26.

Verbree, E., Zlatanova, S., Van Winden, K., Van Der Laan, E., Makri, A., Taizhou, L., Haojun, A., 2013. To localise or to be localised with WiFi in the Hubei museum? Int. Arch. Photogramm. Remote Sens. Spat. Inf. Sci. - ISPRS Arch. 40, 31-35.

Wang, L., Sohn, G., 2011. An Integrated Framework for Reconstructing Full 3D Building Models. Lect. Notes Geoinf. Cartogr. XXXVIII, 261-274.

Werner, M., 2014. Indoor Location-Based Services. Springer International Publishing, Cham.

Wohlfeil, J., Strackenbrock, B., Kossyk, I., 2013. Automated high resolution $3 \mathrm{~d}$ reconstruction of cultural heritage using multi-scale sensor systems and semi-global matching. Int. Arch. Photogramm. Remote Sens. Spat. Inf. Sci. - ISPRS Arch. 40, 37-43.

Xiong, Q., Zhu, Q., Zlatanova, S., Du, Z., Zhang, Y., Zeng, L., 2015. Multi-Level Indoor Path Planning Method. ISPRS - Int. Arch. Photogramm. Remote Sens. Spat. Inf. Sci. XL-4/W5, 1923.

Yokoi, K., Yabuki, N., Fukuda, T., Michikawa, T., Motamedi, A., 2015. Way-Finding Assistance System for Underground Facilities Using Augmented Reality. ISPRS - Int. Arch. Photogramm. Remote Sens. Spat. Inf. Sci. XL-4/W5, 37-41.

Yoon, S., Jung, J., Heo, J., 2015. Practical Implementation of Semi-Automated As-Built Bim Creation for Complex Indoor Environments. ISPRS - Int. Arch. Photogramm. Remote Sens. Spat. Inf. Sci. XL-4/W5, 143-146. 